\title{
The energy cascade at the turbulent/non-turbulent interface
}

\author{
Y. Zhou ${ }^{1}$ and J. C. Vassilicos² \\ ${ }^{1}$ School of Energy and Power Engineering, Nanjing University of Science and Technology, Nanjing 210094, China \\ ${ }^{2}$ Univ. Lille, CNRS, ONERA, Arts et Metiers Institute of Technology, Centrale Lille, \\ UMR 9014 - LMFL - Laboratoire de Mécanique des fluides de Lille - Kampé de Feriet, F-59000 Lille, France
}

(Dated: April 7, 2020)

\begin{abstract}
Interscale energy transfers at the vicinity of the turbulent/non-turbulent interface are from small to large scales in directions close to the interface's tangent plane where motions are predominantly stretching, but from large to small scales in the other directions where motions are predominantly compressive and significantly correlated with square angular momentum. An important role in this predominance is played by the extreme compressive motions which can be significantly more likely than extreme stretching motions even where motions are on average stretching. The most intense interscale transfer rates and dissipation occur when the interface is as far as possible from the wake centreline.
\end{abstract}

Typically for statistical physics, the number of degrees of freedom in fully resolved Navier-Stokes simulations of turbulent flows is prohibitively large at high Reynolds numbers (ratio of inertial to viscous forces). The turbulence problem is to reliably reduce the number of degrees of freedom, either universally or in different ways in different universality classes. This reduced order modeling requires understanding of the non-linear physics which determine turbulence dynamics and statistics.

An important feature of many environmental, geophysical and industrial turbulent shear flows (turbulent wakes, jets, boundary/mixing layers, etc) of very wide relevance, including for mixing and cloud physics [1, 2], is the presence of a sharp interface between potential non-turbulent flow and vortical turbulent flow (first studied systematically by Corrsin and Kistler [3]). These Turbulent/Non-Turbulent Interfaces (TNTI) pose a serious challenge to reduced order modeling because they are conceptually hard to reconcile with eddy viscosities [4] and they incorporate a wide range of scales of motion. Intimately linked to this wide range of scales which characterise turbulent flows is the energy cascade which is a direct consequence of non-linearity at high Reynolds numbers. To the authors's knowledge, nothing is known about the energy cascade and interscale energy transfers at the TNTI. Are these interscale transfers from large to small or from small to large scales, and do they depend on orientation relative to the TNTI? How do they correlate with local rotation, local stretching and compression, and perhaps also other non-linear processes such as turbulent transport? Do interscale transfers and turbulence dissipation depend on TNTI location? This letter offers answers to these questions which concern essential turbulence physics of direct relevance to reduced order modeling, in particular coarse-grained representations of turbulence and related subgrid scale modeling for Large Eddy Simulations where interscale energy transfer is key.

We use data from a state-of-the-art massively parallel Direct Numerical Simulation (DNS) of spatially evolv-

\footnotetext{
* john-christos.vassilicos@centralelille.fr
}

ing axisymmetric and incompressible turbulent wake obtained by Dairay et al. [5]. In terms of free stream velocity $U_{\infty}$ and area $L_{b}^{2}$ of the bluff plate placed normal to the incoming laminar free stream, the Reynolds number is $U_{\infty} L_{b} / \nu=5000$ (fluid's kinematic viscosity $\nu$ ). The computational domain is long $\left(120 L_{b}\right)$ and data were reliably collected up to a distance $x=100 L_{b}$ from the plate. The spatial resolution increases with $x / L_{b}$ and equals the Kolmogorov microscale $\eta_{K}$ at $x=60 L_{b}$ where our study's results were obtained. At $x=60 L_{b}$ on the centreline, $\eta_{K} \approx 0.025 L_{b}$ and the Taylor and longitudinal integral length scales are $\lambda \approx 0.37 L_{b}$ and $L \approx 1.97 L_{b}$.

We calculate statistics conditional on TNTI location within the $x=60 L_{b}$ plane. We locate the TNTI in the way that Zhou and Vassilicos [6] located it for the same DNS data set, by computing the instantaneous area $A_{t}$ within the $x=60 L_{b}$ plane where the modulus $\omega$ of the instantaneous enstrophy exceeds a threshold $\omega_{t h}$. $A_{t}$ decreases with increasing $\omega_{t h}$, reaching zero at $\omega_{t h}=\omega_{\max }(t)$, the maximum $\omega$ in the $x=60 L_{b}$ plane at time $t$. The presence of the TNTI manifests itself by a plateau in the plot of $A_{t}$ versus $\omega_{t h} / \omega_{\max }$ (this plot is similar whether $\omega_{t h} / \omega_{\max }$ is kept constant in time or not), see figure 1 in Zhou and Vassilicos [6]. At $x=60 L_{b}$, $A_{t}$ is about constant over the $\omega_{t h} / \omega_{\max }$ range from about $2.5 \times 10^{-4}$ to $10^{-2}$. This wide vorticity range is taversed over a thin region of space, the TNTI, which explains the wide plateau in the $A_{t}$ versus $\omega_{t h} / \omega_{\max }$ plot. In this letter, we chose $\omega_{t h} / \omega_{\max }=4 \times 10^{-4}$ to locate points on the TNTI in the $x=60 L_{b}$ plane.

To answer our questions on interscale energy transfers at the TNTI we need a scale-by-scale energy budget that is local in space and time. This budget is the fully generalised Kármán-Howarth equation, i.e. the version of the Kármán-Howarth-Monin-Hill (KHMH) equation directly derived from the incompressible Navier-Stokes equations for the instantaneous velocity field [7, 8] without Reynolds or other decomposition, without averaging operations, and without assumptions about the turbulence. This is the evolution equation for $|\delta \boldsymbol{u}|^{2}$, where $\delta \boldsymbol{u} \equiv \boldsymbol{u}-\boldsymbol{u}^{\prime}$ is the difference of fluid velocities between points $\boldsymbol{x}$ and $\boldsymbol{x}^{\prime}, \boldsymbol{u} \equiv \boldsymbol{u}(\boldsymbol{x}, t)$ and $\boldsymbol{u}^{\prime} \equiv \boldsymbol{u}\left(\boldsymbol{x}^{\prime}, t\right)$. It is ex- 
pressed in terms of centroid $\boldsymbol{X}=\left(\boldsymbol{x}+\boldsymbol{x}^{\prime}\right) / 2$, separation vector $\boldsymbol{r}=\boldsymbol{x}-\boldsymbol{x}^{\prime}$ and time $t$ as follows:

$$
\begin{aligned}
\frac{\partial}{\partial t}|\delta \boldsymbol{u}|^{2} & +\frac{\partial}{\partial r_{k}}\left(\delta u_{k}|\delta \boldsymbol{u}|^{2}\right)=-\frac{\partial}{\partial X_{k}} \frac{\left(u_{k}+u_{k}^{\prime}\right)|\delta \boldsymbol{u}|^{2}}{2} \\
& -\frac{2}{\rho} \frac{\partial}{\partial X_{k}}\left(\delta u_{k} \delta p\right)+2 \nu \frac{\partial^{2}}{\partial r_{k}^{2}}|\delta \boldsymbol{u}|^{2}+\frac{\nu}{2} \frac{\partial^{2}}{\partial X_{k}^{2}}|\delta \boldsymbol{u}|^{2} \\
& -\left[2 \nu\left(\frac{\partial u_{j}}{\partial x_{k}}\right)^{2}+2 \nu\left(\frac{\partial u_{j}^{\prime}}{\partial x_{k}^{\prime}}\right)^{2}\right]
\end{aligned}
$$

where $\rho$ is fluid density and $\delta p=p(\boldsymbol{x})-p\left(\boldsymbol{x}^{\prime}\right)$ is the pressure difference across the two points.

We define an average energy over scales smaller than $r=|\boldsymbol{r}|$ as $E(\boldsymbol{X}, r, t)=\frac{3}{\pi r^{3}} \int_{V(\boldsymbol{X}, r)} d^{3} \boldsymbol{r}|\delta \boldsymbol{u}|^{2}$ where the integral is over the volume $V(\boldsymbol{X}, r)$ of a sphere of diameter $r$ centred at $\boldsymbol{X} . E(\boldsymbol{X}, r, t)$ typically varies as $r^{2}$ for $r \leq O\left(\eta_{K}\right)$ and oscillates around a constant when $r$ is so large that fluid velocities at $\boldsymbol{x}$ and $\boldsymbol{x}^{\prime}$ fluctuate independently. The evolution equation for $E(\boldsymbol{X}, r, t)$ is obtained by applying operation $\frac{3}{\pi r^{3}} \int_{V(\boldsymbol{X}, r)} d^{3} \boldsymbol{r}$ to all terms in equation (1), and the left side of this evolution equation is $\frac{\partial}{\partial t} E+\frac{3}{\pi r^{3}} \int_{V(\boldsymbol{X}, r)} d^{3} \boldsymbol{r} \frac{\partial}{\partial r_{k}}\left(\delta u_{k}|\delta \boldsymbol{u}|^{2}\right)$. Using the divergence theorem, this left side's second term is proportional to a scale-space flux because $\int_{V(\boldsymbol{X}, r)} d^{3} \boldsymbol{r} \frac{\partial}{\partial r_{k}}\left(\delta u_{k}|\delta \boldsymbol{u}|^{2}\right)=$ $\int_{\partial V(\boldsymbol{X}, r)} d^{2} \boldsymbol{r} \delta \boldsymbol{u} \cdot \hat{\boldsymbol{r}}|\delta \boldsymbol{u}|^{2}$, where $\int_{\partial V(\boldsymbol{X}, r)} d^{2} \boldsymbol{r}$ is an integral over the surface of the sphere of diameter $r$ centred at $\boldsymbol{X}$ and $\hat{\boldsymbol{r}} \equiv \boldsymbol{r} / r$. Simplifying further, the left side becomes $\frac{\partial}{\partial t} E+\frac{3}{\pi} \int d \Omega \frac{\delta \boldsymbol{u} \cdot \hat{r}}{r}|\delta \boldsymbol{u}|^{2}$ where $\Omega$ is the solid angle. The interscale transfer rate $\frac{3}{\pi} \int d \Omega \frac{\delta \boldsymbol{u} \cdot \hat{r}}{r}|\delta \boldsymbol{u}|^{2}$ (space-scale flux if multiplied by $r^{3}$ ) vanishes at $r=0$ and tends to 0 as $r$ grows indefinitely. At a given finite scale $r$, a scale-space flux from large to small or from small to large scales corresponds to a negative or positive $\frac{3}{\pi} \int d \Omega \frac{\delta \boldsymbol{u} \cdot \hat{\boldsymbol{r}}}{r}|\delta \boldsymbol{u}|^{2}$ and contributes a growth or decrease of $E(\boldsymbol{X}, r, t)$ in time. In highly inhomogeneous/anisotropic flows, particularly in the vicinity of the TNTI, the interscale transfer rate does not necessarily dominate the behaviour of $E(\boldsymbol{X}, r, t)$ as the terms on the right side of the equation involve a pressure-velocity term $-\frac{\partial}{\partial X_{k}} \frac{3}{\pi r^{3}} \int_{V(\boldsymbol{X}, r)} d^{3} \boldsymbol{r} \frac{2}{\rho}\left(\delta u_{k} \delta p\right)$ and a term $-\frac{\partial}{\partial X_{k}} \frac{3}{\pi r^{3}} \int_{V(\boldsymbol{X}, r)} d^{3} \boldsymbol{r} \frac{\left(u_{k}+u_{k}^{\prime}\right)|\delta \boldsymbol{u}|^{2}}{2}$, which may dominate. This latter term includes mean advection and production terms as well as the spatial turbulent transfer rate $-\frac{\partial}{\partial X_{k}} \frac{3}{\pi r^{3}} \int_{V(\boldsymbol{X}, r)} d^{3} \boldsymbol{r} \frac{\left(u_{k}-U_{k}+u_{k}^{\prime}-U_{k}^{\prime}\right)|\delta \boldsymbol{u}|^{2}}{2}$ where $U_{k}$ and $U_{k}^{\prime}$ are mean flow velocity components obtained by averaging over time at $\boldsymbol{x}$ and $\boldsymbol{x}^{\prime}$ respectively.

A forward space-scale flux from large to small scales corresponds to predominance of compression, $\delta \boldsymbol{u} \cdot \hat{\boldsymbol{r}}<0$, so that $\int d \Omega \frac{\delta \boldsymbol{u} \cdot \hat{\boldsymbol{r}}}{r}|\delta \boldsymbol{u}|^{2}$ is negative. Conversely, space-scale flux from small to large scales, corresponds to predominance of stretching, $\delta \boldsymbol{u} \cdot \hat{\boldsymbol{r}}>0$, so that $\int d \Omega \frac{\delta \boldsymbol{u} \cdot \hat{r}}{r}|\delta \boldsymbol{u}|^{2}$ is positive. Incompressibility implies $\int d \Omega \delta \boldsymbol{u} \cdot \hat{\boldsymbol{r}}=0$. As fluid elements approach the TNTI without vorticity and obtain vorticity by crossing it, their velocity normal to the TNTI changes depending on reorientation of their motion (which suddenly becomes vortical) and on a sudden decrease in pressure [4, [9]. If this normal velocity change helps sustain the TNTI, one may expect $\delta \boldsymbol{u} \cdot \hat{\boldsymbol{r}}$ to be negative for $\hat{\boldsymbol{r}}$ around the normal to the TNTI, and by virtue of $\int d \Omega \delta \boldsymbol{u} \cdot \hat{\boldsymbol{r}}=0$, one may also expect $\delta \boldsymbol{u} \cdot \hat{\boldsymbol{r}}$ to be positive for $\hat{\boldsymbol{r}}$ close to tangent to the TNTI. Indeed, compressive relative motions in the direction normal to the TNTI and stretching relative motions in directions tangent to the TNTI sustain the TNTI. The question arises whether the interscale transfers at the TNTI reflect a TNTI self-sustaining mechanism so that $\delta \boldsymbol{u} \cdot \hat{\boldsymbol{r}}|\delta \boldsymbol{u}|^{2}<0$ for $\hat{\boldsymbol{r}}$ in the vicinity of the TNTI's normal and $\delta \boldsymbol{u} \cdot \hat{\boldsymbol{r}}|\delta \boldsymbol{u}|^{2}>0$ for $\hat{\boldsymbol{r}}$ in the vicinity of the TNTI's tangent plane. The question which follows is to know the sign of the resulting aggregate interscale transfer rate $\int d \Omega \frac{\delta \boldsymbol{u} \cdot \hat{\boldsymbol{r}}}{r}|\delta \boldsymbol{u}|^{2}$ at the TNTI.

To answer these questions, we start with identifying those numerical mesh points in the $x=60 L_{b}$ plane where $\omega$ is closest to $\omega_{t h}$. These points are on the TNTI. (Details of the procedure to locate the TNTI are in [6].) We compute $\boldsymbol{n}=\boldsymbol{\nabla} \omega^{2} /\left|\nabla \omega^{2}\right|$, the unit vector normal to the TNTI in 3D space, and select those particular points $\boldsymbol{X}_{I}$ on the TNTI where $\omega\left(\boldsymbol{X}_{I}+c L_{b} \boldsymbol{n}\right)>\omega_{t h}$ and $\omega\left(\boldsymbol{X}_{I}-c L_{b} \boldsymbol{n}\right)<\omega_{t h}$ for $0<c<1$. These are the points on the interface which do not face folds over scales smaller than about $2 / 3$ of the wake width $\delta(x)$ at $x=60 L_{b}$ (given that $\delta \approx 1.55 L_{b}$ at $x=60 L_{b}$ ). We therefore limit our study to those points on the TNTI where folds do not contribute to the TNTI's interscale transfer properties. With our very strict criterion $0<c<1$, we consider about half the points on the TNTI, but this proportion is much larger for a smaller range of positive values of $c$, in which case our analysis can be expected to carry over up to smaller maximum values of $r$.

We then compute the angle $\theta_{r}$ between $\boldsymbol{n}$ and $\boldsymbol{r}$ $\left(\cos \theta_{r}=\boldsymbol{n} \cdot \hat{\boldsymbol{r}}\right)$ and an angle $\phi_{r}$ which locates the projection of $\boldsymbol{r}$ on the local tangent plane normal to $\boldsymbol{n}$. Finally we calculate the conditional averages $\left\langle\delta \boldsymbol{u} \cdot \hat{\boldsymbol{r}}|\delta \boldsymbol{u}|^{2} \mid \boldsymbol{X}_{I}\right\rangle$ and $\left\langle\delta \boldsymbol{u} \cdot \hat{\boldsymbol{r}} \mid \boldsymbol{X}_{I}\right\rangle$ by averaging over 375 randomly selected instantaneous velocity fields from 15000 time steps and over $\phi_{r}$. These averages are conditioned on the TNTI points $\boldsymbol{X}_{I}$ and are plotted in figure 1a,b as functions of $r_{n} / \delta$ and $r_{t} / \delta$ where $r_{n}=\boldsymbol{n} \cdot \boldsymbol{r}$ and $r_{t}^{2}=r^{2}-r_{n}^{2}\left(\tan \theta_{r}=r_{t} / r_{n}\right)$. The results in all our figures (except figure $2 b$ ), stay very similar if the fluid velocity $\boldsymbol{u}$ is replaced by the fluctuating velocity $\boldsymbol{u}-\boldsymbol{U}$ where $\boldsymbol{U}$ is the mean flow velocity obtained by averaging over time.

Figure 1a,b shows that $\left\langle\delta \boldsymbol{u} \cdot \hat{\boldsymbol{r}}|\delta \boldsymbol{u}|^{2} \mid \boldsymbol{X}_{I}\right\rangle$ and $\left\langle\delta \boldsymbol{u} \cdot \hat{\boldsymbol{r}} \mid \boldsymbol{X}_{I}\right\rangle$ are both negative above the magenta line, i.e. for angles $\theta_{r}$ below about $50^{\circ}$, and both positive below the black line in figure $1 \mathrm{~b}$, i.e. for angles $\theta_{r}$ above about $70^{\circ}-75^{\circ}$. In this average sense, the interscale transfers at the selected TNTI points seem to be a reflection of compressive motions for $\boldsymbol{r}$ more or less aligned with the normal to the TNTI and a reflection of stretching motions in directions more or less aligned with the TNTI's tangent plane at points $\boldsymbol{X}_{I}$. Because of these compressive and stretching motions which sustain the TNTI, interscale transfers in the range $r_{n} / \delta<1.0$ and $r_{t} / \delta<1.0$ are from large to 

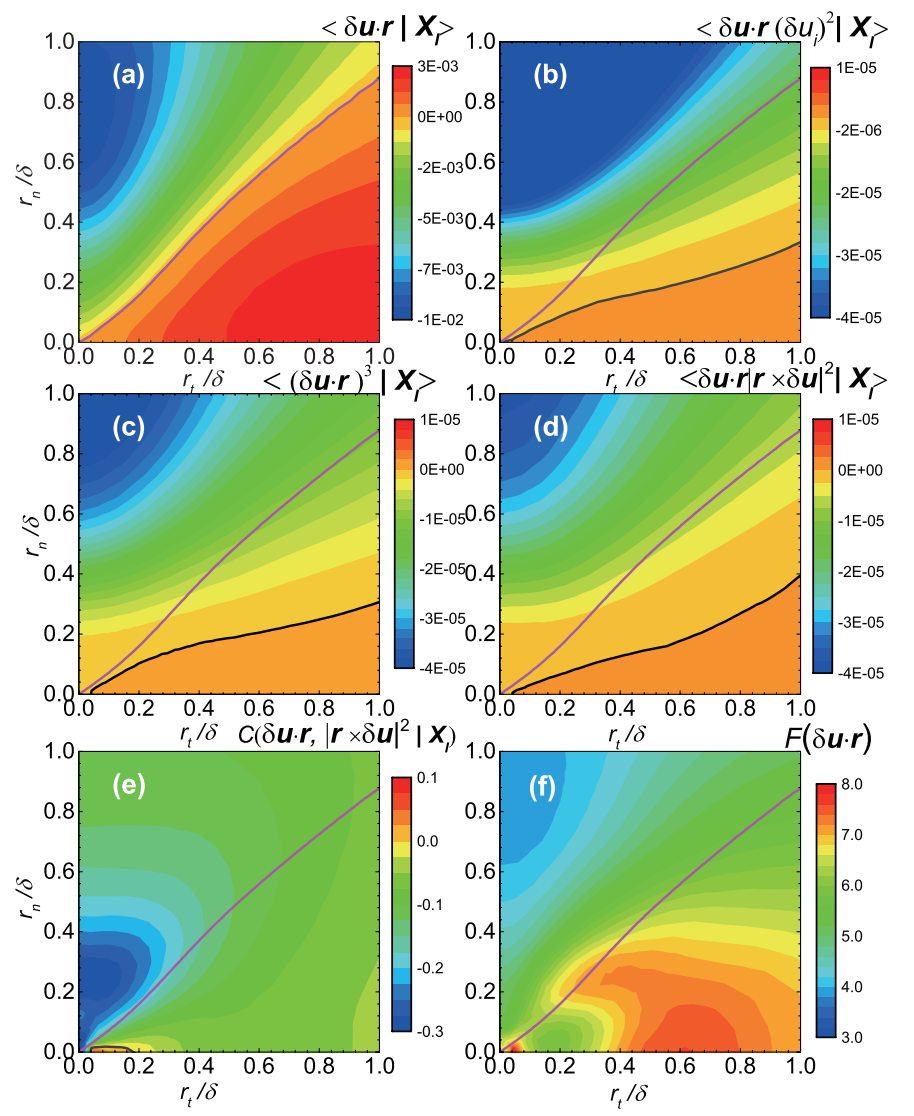

FIG. 1. Plots in the $\left(r_{n} / \delta, r_{t} / \delta\right)$ plane (wake width $\delta$ at $x / L_{b}=60$ ) of (a) $\left\langle\delta \boldsymbol{u} \cdot \hat{\boldsymbol{r}} \mid \boldsymbol{X}_{I}\right\rangle$, (b) $\left\langle\delta \boldsymbol{u} \cdot \hat{\boldsymbol{r}}|\delta \boldsymbol{u}|^{2} \mid \boldsymbol{X}_{I}\right\rangle$, (c) $\langle(\delta \boldsymbol{u}$. $\hat{\boldsymbol{r}})^{3}\left|\boldsymbol{X}_{I}\right\rangle$, (d) $\left\langle\delta \boldsymbol{u} \cdot \hat{\boldsymbol{r}}|\hat{\boldsymbol{r}} \times \delta \boldsymbol{u}|^{2} \mid \boldsymbol{X}_{I}\right\rangle$, (e) the Pearson correlation coefficient $C$ between $\delta \boldsymbol{u} \cdot \hat{\boldsymbol{r}}$ and $|\hat{\boldsymbol{r}} \times \delta \boldsymbol{u}|^{2}$ conditional on $\boldsymbol{X}_{I}$ and (f) the flatness of $\delta \boldsymbol{u} \cdot \hat{\boldsymbol{r}}$ conditional on $\boldsymbol{X}_{I}$. The magenta line in plots (a) to (f) is where $\left\langle\delta \boldsymbol{u} \cdot \hat{\boldsymbol{r}} \mid \boldsymbol{X}_{I}\right\rangle=0$. The black line in plots (b) to (e) is where the quantity plotted is 0 .

small scales for angles $\theta_{r}$ below about $50^{\circ}$ but from small to large scales for angles $\theta_{r}$ above about $70^{\circ}-75^{\circ}$.

Surprisingly, in the range of angles $\theta_{r}$ between about $50^{\circ}$ and about $70^{\circ}-75^{\circ}$ where the motions are stretching on average, i.e. $\left\langle\delta \boldsymbol{u} \cdot \hat{\boldsymbol{r}} \mid \boldsymbol{X}_{I}\right\rangle$ is positive, the interscale transfer is on average "compressive", i.e. $\left\langle\delta \boldsymbol{u} \cdot \hat{\boldsymbol{r}}|\delta \boldsymbol{u}|^{2} \mid \boldsymbol{X}_{I}\right\rangle$ is negative. To better understand this intermediate range of angles where interscale transfer is from large to small scales even though fluid element pairs tend to separate on average, we use the decomposition

$$
\left\langle\delta \boldsymbol{u} \cdot \hat{\boldsymbol{r}}|\delta \boldsymbol{u}|^{2} \mid \boldsymbol{X}_{I}\right\rangle=\left\langle(\delta \boldsymbol{u} \cdot \hat{\boldsymbol{r}})^{3} \mid \boldsymbol{X}_{I}\right\rangle+\left\langle\delta \boldsymbol{u} \cdot \hat{\boldsymbol{r}}|\hat{\boldsymbol{r}} \times \delta \boldsymbol{u}|^{2} \mid \boldsymbol{X}_{I}\right\rangle
$$

which shows that $\left\langle\delta \boldsymbol{u} \cdot \hat{\boldsymbol{r}}|\delta \boldsymbol{u}|^{2} \mid \boldsymbol{X}_{I}\right\rangle$ can indeed be negative when $\left\langle\delta \boldsymbol{u} \cdot \hat{\boldsymbol{r}} \mid \boldsymbol{X}_{I}\right\rangle$ is positive if $\left\langle(\delta \boldsymbol{u} \cdot \hat{\boldsymbol{r}})^{3} \mid \boldsymbol{X}_{I}\right\rangle$ is negative enough or if $\left\langle\delta \boldsymbol{u} \cdot \hat{\boldsymbol{r}}|\hat{\boldsymbol{r}} \times \delta \boldsymbol{u}|^{2} \mid \boldsymbol{X}_{I}\right\rangle$ is negative enough or if both are negative. This decomposition states that the interscale energy transfer consists of a transfer of longitudinal energy and a transfer of rotational energy.

Figure 1c shows that $\left\langle(\delta \boldsymbol{u} \cdot \hat{\boldsymbol{r}})^{3} \mid \boldsymbol{X}_{I}\right\rangle$ has the same sign as $\left\langle\delta \boldsymbol{u} \cdot \hat{\boldsymbol{r}}|\delta \boldsymbol{u}|^{2} \mid \boldsymbol{X}_{I}\right\rangle$ effectively everywhere in the $\left(r_{n}, r_{t}\right)$ plane. However, figure $1 \mathrm{~d}$ shows the same for $\left\langle\delta \boldsymbol{u} \cdot \hat{\boldsymbol{r}}|\hat{\boldsymbol{r}} \times \delta \boldsymbol{u}|^{2} \mid \boldsymbol{X}_{I}\right\rangle$. Furthermore, these three different conditional statistics have comparable magnitudes effectively everywhere in the $\left(r_{n}, r_{t}\right)$ plane. To explain the sign and magnitude of the local interscale transfer rate $\left\langle\frac{\delta \boldsymbol{u} \cdot \hat{\boldsymbol{r}}}{r}|\delta \boldsymbol{u}|^{2} \mid \boldsymbol{X}_{I}\right\rangle$ one therefore needs to take both $\left\langle(\delta \boldsymbol{u} \cdot \hat{\boldsymbol{r}})^{3} \mid \boldsymbol{X}_{I}\right\rangle$ and $\left\langle\delta \boldsymbol{u} \cdot \hat{\boldsymbol{r}}|\hat{\boldsymbol{r}} \times \delta \boldsymbol{u}|^{2} \mid \boldsymbol{X}_{I}\right\rangle$ into account. For this, we use a second decomposition, namely

$$
\begin{array}{r}
\left\langle\delta \boldsymbol{u} \cdot \hat{\boldsymbol{r}}|\hat{\boldsymbol{r}} \times \delta \boldsymbol{u}|^{2} \mid \boldsymbol{X}_{I}\right\rangle= \\
\left\langle\delta \boldsymbol{u} \cdot \hat{\boldsymbol{r}} \mid \boldsymbol{X}_{I}\right\rangle\left\langle|\hat{\boldsymbol{r}} \times \delta \boldsymbol{u}|^{2} \mid \boldsymbol{X}_{I}\right\rangle+C \sigma_{C S} \sigma_{L}
\end{array}
$$

where $\sigma_{C S}$ and $\sigma_{L}$ are standard deviations (conditional on $\boldsymbol{X}_{I}$ ) of $\delta \boldsymbol{u} \cdot \hat{\boldsymbol{r}}$ and $|\hat{\boldsymbol{r}} \times \delta \boldsymbol{u}|^{2}$ respectively, and $C$ is the Pearson correlation coefficient conditional on $\boldsymbol{X}_{I}$ between compression/stretching relative velocity $\delta \boldsymbol{u} \cdot \hat{\boldsymbol{r}}$ and $|\boldsymbol{L}|^{2}, \boldsymbol{L} \equiv \frac{1}{2} \boldsymbol{r} \times \delta \boldsymbol{u}$ being the angular momentum per unit mass of the fluid elements at $\boldsymbol{x}$ and $\boldsymbol{x}^{\prime}$ with respect to the centroid $\boldsymbol{X}$. The plot of $C$ (figure 1e) shows a small but significant negative correlation between $\delta \boldsymbol{u} \cdot \hat{\boldsymbol{r}}$ and $|\boldsymbol{L}|^{2}$ nearly everywhere in the $\left(r_{n}, r_{t}\right)$ plane (except in a small region along the $r_{t} / \delta$ axis). Compression at the TNTI has some positive correlation with the square of the angular momentum relative to the TNTI, particularly for orientations of $\boldsymbol{r}$ normal to the TNTI and up to about $45^{\circ}$ to that normal, and particularly for $r / \delta$ smaller than about 0.4 .

We can now explain the sign of the local interscale transfer at points $\boldsymbol{X}_{I}$ on the TNTI. From equations (2)(3), this sign is determined by the signs of $\left\langle(\delta \boldsymbol{u} \cdot \hat{\boldsymbol{r}})^{3} \mid \boldsymbol{X}_{I}\right\rangle$, $\left\langle\delta \boldsymbol{u} \cdot \hat{\boldsymbol{r}} \mid \boldsymbol{X}_{I}\right\rangle$ and $C$. In the $\left(r_{n}, r_{t}\right)$ plane region where $\theta_{r}$ is below about $50^{\circ}$, all these signs are negative (figure 1a,c,e). Consequently, the interscale transfer at our selected TNTI points $\boldsymbol{X}_{I}$ is from large to small scales for angles up to about $50^{\circ}$ to the TNTI's normal (figure 1b) because of the predominantly compressive motions and because of the significant correlation of these compressive motions with angular momentum at these angles. The compressive motions contribute via negative values of both $\left\langle(\delta \boldsymbol{u} \cdot \hat{\boldsymbol{r}})^{3} \mid \boldsymbol{X}_{I}\right\rangle$ and $\left\langle\delta \boldsymbol{u} \cdot \hat{\boldsymbol{r}} \mid \boldsymbol{X}_{I}\right\rangle$ at these orientations, meaning that extreme compressive motions are significantly more likely than extreme stretching motions and that motions are also compressive on average. The presence of extreme events is confirmed by figure $1 \mathrm{f}$ which shows that the flatness of $\delta \boldsymbol{u} \cdot \hat{\boldsymbol{r}}$ conditional on TNTI points $\boldsymbol{X}_{I}$ is larger than about 4 throughout the $\left(r_{n}, r_{t}\right)$ plane and increases up to values close to 8 with increasing $\theta_{r}$. In the intermediate range of angles $\theta_{r}$ from about $50^{\circ}$ to about $70^{\circ}-75^{\circ}$, the motions become stretching on average, i.e. $\left\langle\delta \boldsymbol{u} \cdot \hat{\boldsymbol{r}} \mid \boldsymbol{X}_{I}\right\rangle$ becomes positive (figure 1a), but remain negatively skewed, i.e. $\left\langle(\delta \boldsymbol{u} \cdot \hat{\boldsymbol{r}})^{3} \mid \boldsymbol{X}_{I}\right\rangle$ remains negative (figure 1c). In this range of angles, the interscale transfer at the selected TNTI points $\boldsymbol{X}_{I}$ remains from large to small scales (figure 1b) and the compressive motions remain responsible for this forward transfer but in a different way. It is now the fact that extreme compressive motions are significantly more likely than extreme stretching motions and that the compressive motions remain correlated with angular momentum which keeps this interscale transfer flowing from large to 


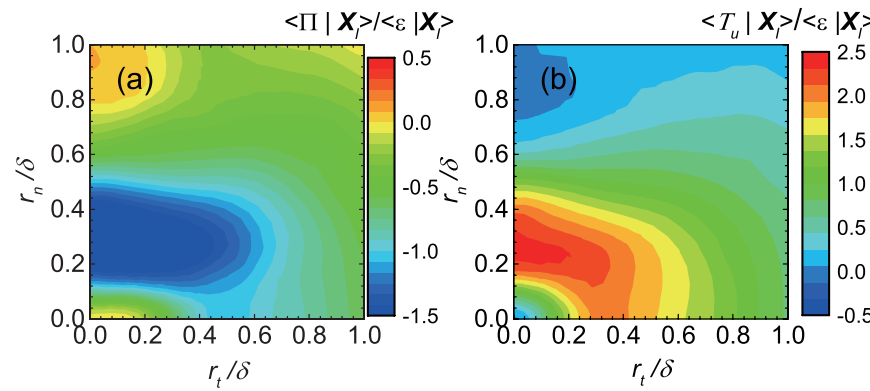

FIG. 2. Plots in the $\left(r_{n} / \delta, r_{t} / \delta\right)$ plane (wake width $\delta$ at $\left.x / L_{b}=60\right)$ of (a) $\left\langle\Pi \mid \boldsymbol{X}_{I}\right\rangle /\left\langle\varepsilon_{r} \mid \boldsymbol{X}_{I}\right\rangle$ and (b) $\left\langle T_{u} \mid \boldsymbol{X}_{I}\right\rangle /\left\langle\varepsilon_{r} \mid \boldsymbol{X}_{I}\right\rangle$.

small scales. The negative values that these two effects contribute to $\left\langle\delta \boldsymbol{u} \cdot \hat{\boldsymbol{r}}|\delta \boldsymbol{u}|^{2} \mid \boldsymbol{X}_{I}\right\rangle$ (equations (2)-(3)) overcome the positive value of $\left\langle\delta \boldsymbol{u} \cdot \hat{\boldsymbol{r}} \mid \boldsymbol{X}_{I}\right\rangle\left\langle|\hat{\boldsymbol{r}} \times \delta \boldsymbol{u}|^{2} \mid \boldsymbol{X}_{I}\right\rangle$ in this intermediate range of angles $\theta_{r}$ where the motions are now stretching on average.

As the angle $\theta_{r}$ grows beyond $70^{\circ}-75^{\circ}$, the motions become predominantly stretching both on average and also in the sense that extreme stretching events become more likely than extreme compressive events (figure 1a,c). Furthermore the correlation between compressive motions and angular momentum is weaker in the vicinity of the TNTI's tangent plane. In fact, $C$ does not only take weaker negative values but even small positive ones for some values of $r_{n}$ and $r_{t}$ (figure 1e). The result (figure 1b) is a small positive $\left\langle\delta \boldsymbol{u} \cdot \hat{\boldsymbol{r}}|\delta \boldsymbol{u}|^{2} \mid \boldsymbol{X}_{I}\right\rangle$ (equations (2)(3)) and therefore a weak average interscale backscatter at these orientations.

The next question concerns the sign of the timeaverage conditional on $\boldsymbol{X}_{I}$ of the resulting aggregate interscale transfer rate $\frac{3}{\pi} \int d \Omega \frac{\delta \boldsymbol{u} \cdot \boldsymbol{r}}{r}|\delta \boldsymbol{u}|^{2}$ at the TNTI. This conditional time-average is $12 \int_{0}^{\pi / 2} d \theta_{r} \sin \theta_{r}\left\langle\frac{\delta \boldsymbol{u} \cdot \hat{\boldsymbol{r}}}{r}|\delta \boldsymbol{u}|^{2} \mid \boldsymbol{X}_{I}\right\rangle \quad$ and it equals $\frac{12}{r^{3}} \int_{0}^{r} d r r^{2} \int_{0}^{\pi / 2} d \theta_{r} \sin \theta_{r}\left\langle\Pi \mid \boldsymbol{X}_{I}\right\rangle$ where $\Pi \equiv \Pi(\boldsymbol{X}, \boldsymbol{r}) \equiv$ $\frac{\partial}{\partial r_{k}}\left(\delta u_{k}|\delta \boldsymbol{u}|^{2}\right)$. In figure $2 \mathrm{a}$, the plot of $\left\langle\Pi \mid \boldsymbol{X}_{I}\right\rangle /\left\langle\varepsilon_{r} \mid \boldsymbol{X}_{I}\right\rangle$ in the $\left(r_{n}, r_{t}\right)$ plane $\left(2 \varepsilon_{r}\right.$ is the sum of the kinetic energy dissipation rates at $\boldsymbol{x}$ and $\boldsymbol{x}^{\prime}$ ) shows that $\left\langle\Pi \mid \boldsymbol{X}_{I}\right\rangle$ is negative everywhere within $0 \leq r_{n} / \delta \leq 1.0$ and $0 \leq r_{t} / \delta \leq 1.0$ except in a small region at the top left of this region of the $\left(r_{n}, r_{t}\right)$ plane where it takes small positive values. Clearly, $\int_{0}^{\pi / 2} d \theta_{r} \sin \theta_{r}\left\langle\Pi \mid \boldsymbol{X}_{I}\right\rangle$ is negative for all $r / \delta \leq 1.0$ : inspite of the combined forward and backscatter interscale transfers at different orientations with respect of the TNTI, the resulting aggregate interscale transfer at the TNTI is from large to small scales for all scales $r \leq \delta$.

Spatial energy transfers turn out to be as, if not even more, important than the interscale transfers at the TNTI, and to even correlate with them in the $\left(r_{n}, r_{t}\right)$ plane. Compare the plot of $\left\langle\Pi \mid \boldsymbol{X}_{I}\right\rangle /\left\langle\varepsilon_{r} \mid \boldsymbol{X}_{I}\right\rangle$ in figure 2a with the plot of $\left\langle T_{u} \mid \boldsymbol{X}_{I}\right\rangle /\left\langle\varepsilon_{r} \mid \boldsymbol{X}_{I}\right\rangle$ in figure $2 \mathrm{~b}$, where $T_{u} \equiv-\frac{\partial}{\partial X_{k}} \frac{\left(u_{k}-U_{k}+u_{k}^{\prime}-U_{k}^{\prime}\right)|\delta \boldsymbol{u}|^{2}}{2}$ is the spatial non-linear energy transport rate in equation (1). The scales and ori- entations where the local interscale transfer rate takes its highest positive values correspond to those where the local spatial transfer rate takes its highest negative values. These scales $\left(r_{n}\right.$ in particular) are comparable to the centreline Taylor length scale $\lambda\left(\approx 0.24 \delta\right.$ at $\left.x=60 L_{b}\right)$. Furthermore, the small upper left corner in the $\left(r_{n}, r_{t}\right)$ plane where $\left\langle\Pi \mid \boldsymbol{X}_{I}\right\rangle$ is positive (and small) is also the only region in this plane where $\left\langle T_{u} \mid \boldsymbol{X}_{I}\right\rangle$ is negative (and small). The magniture of $\left\langle T_{u} \mid \boldsymbol{X}_{I}\right\rangle$ is typically about twice the magnitude of $\left\langle\Pi \mid \boldsymbol{X}_{I}\right\rangle$. Both interscale and spatial energy transfers result from the Navier-Stokes convective non-linearity and this must be the root cause of their correlation. However future investigations at much higher Reynolds numbers should reveal the extent to which this correlation may be due to the relatively small separation of scales between $\lambda$ and $\delta$ in our DNS data (where the Taylor length-based Reynolds number $R e_{\lambda}=57$ at $x / L_{b}=60$ on the centreline) and/or the extent to which this correlation is an essential part of energy transfers in locally or statistically inhomogeneous situations.

Having analysed interscale transfers in the vicinity of and relative to the TNTI we now investigate whether the position of the TNTI relative to the centreline $y=z=0$ affects interscale transfers and dissipation. We calculate radial TNTI positions $R_{I}(\phi, t)$ in the $(y, z)$ plane at $x=60 L_{b}$ by finding the TNTI's intersections with radial straight lines in this plane which cross the centreline's position $y=z=0$ with an azimuthal angle $\phi$. Relatively rarely, there are more than one intersection between the straight line and the TNTI, in which case the recorded $R_{I}$ value is the largest. We define $\Pi^{a}(\boldsymbol{X}, r, t)=\int d \Omega \Pi$ and consider locations $\boldsymbol{X}$ in the $x=60 L_{b}$ plane. For such locations, $\Pi^{a}(\boldsymbol{X}, r, t)=\Pi^{a}(R, \phi, r, t)$. We focus on $r \approx \lambda$ as the interscale transfer rate is highest near this lengthscale at the TNTI and calculate averages over time and $\phi$ of $\Pi^{a}\left(R, \phi, 0.4 L_{b}, t\right)\left(\lambda \approx 0.38 L_{b}\right.$ at $\left.x=60 L_{b}\right)$, the kinetic energy dissipation rate $\varepsilon(\boldsymbol{X}, t)=\varepsilon(R, \phi, t)$ and $\omega(\boldsymbol{X}, t)=\omega(R, \phi, t)$ conditional on $R_{I}$. These conditional averages (plotted in figure 3 ) are functions of $R$ and $R_{I}$. They all take their largest magnitudes when $R_{I}$ is between $2 \delta$ and $3 \delta$, the furthest distances from the centreline where the TNTI is found. The actual value of $R$ does not seem to matter other than it should not be smaller than about $\delta / 5$ for these three conditional averages to achieve high magnitudes when $R_{I}$ is so large. The high magnitudes of $\Pi^{a}$ are negative, reflecting interscale transfers from large to small scales on average.

Conclusion. The most intense average interscale transfer and the most intense average dissipation and $\omega$ occur when the TNTI is furthest from the centreline and do so more or less uniformly all the way from the TNTI to a finite distance from the centreline. Occurrences of large patches of high enstrophy may simultaneously cause the TNTI to be pushed far from the centreline and interscale transfers to be intense and forward with high dissipation. At the TNTI, interscale transfers are weak and backward in directions close to the TNTI's tangent plane because of straining motions but forward in the other directions 

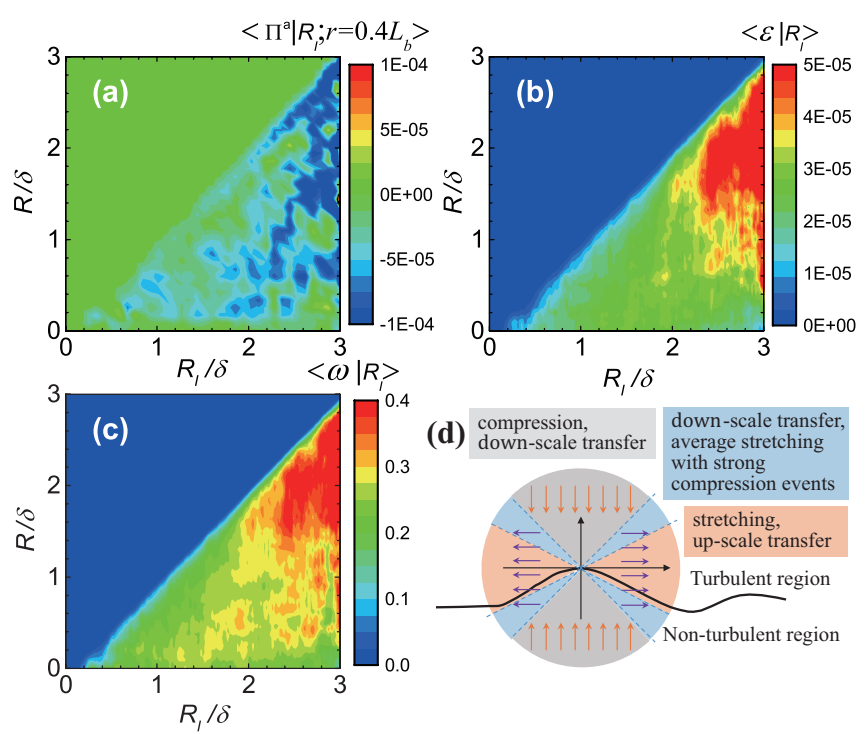

(d) compression, down-scale transfer, (down-scale transfer average stretching

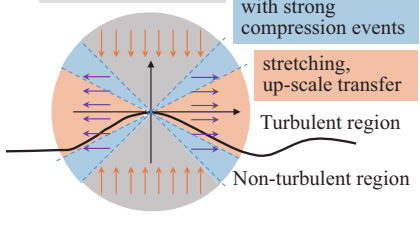

FIG. 3. Averages conditional on $R_{I}$ of (a) $\Pi^{a}$, (b) $\varepsilon$, (c) $\omega$ as functions of $R_{I}$ and $R$ and (d) Summary schematic of the three intercale transfer regions at the TNTI. because of compressive motions. The interscale transfer at the TNTI is forward where extreme compressive motions are more likely than extreme stretching motions even when motions are stretching on average (see summary schematic in figure $3 \mathrm{~d}$ ). A positive correlation exists at the TNTI between compressive motions and angular momentum magnitude. This correlation makes a forward contribution to interscale energy transfers at the TNTI.

\section{ACKNOWLEDGMENTS}

We were supported by the National Natural Science Foundation of China (Nos. 91952105 and 11802133), ERC Advanced Grant 320560 and Chair of Excellence CoPreFlo funded by I-SITE/MEL/Region Hauts de France.
[1] C. B. da Silva, J. C. R. Hunt, I. Eames, and J. Westerweel, Annu. Rev. Fluid Mech. 46, 567 (2014).

[2] J. P. Mellado, Annu. Rev. Fluid Mech. 49, 145 (2016).

[3] S. Corrsin and A. L. Kistler, NACA Technical Report No. TR-1244, 1955, p. 1033.

[4] J. Westerweel, C. Fukushima, J. M. Pedersen, and J. C. R. Hunt, Phys. Rev. Lett. 95, 174501 (2005).
[5] T. Dairay, M. Obligado, and J. C. Vassilicos, J. Fluid Mech. 781, 166 (2015).

[6] Y. Zhou and J. C. Vassilicos, J. Fluid Mech. 821, 440 (2017).

[7] J. Duchon and R. Robert, Nonlinearity 13, 249 (2000).

[8] R. J. Hill, J. Fluid Mech. 468, 317 (2002).

[9] W. C. Reynolds, J. Fluid Mech. 54, 481 (1972). 